\title{
Geschlechtsspezifische Unterschiede in Verlauf und Therapie der chronischen Nierenerkrankung
}

Carrero J], Hecking M, Ulasi I et al. Chronic Kidney Disease, Gender, and Access to Care: A Global Perspective. Semin Nephrol 2017; 37: 296-308

Die geschlechtsspezifischen Unterschiede in der Prävalenz und der Progression der chronischen Nierenerkrankung sind noch wenig erforscht. Noch geringer ist unser Wissen über die geschlechtsspezifischen Unterschiede beim Zugang zur medizinischen Versorgung. Letztlich hängt die Gleichbehandlung der Frau von den aufgewandten Kosten für das Gesundheitswesen und dem gesellschaftlichen System ab.

Der vorliegende Artikel gibt eine Zusammenfassung über die bisher untersuchten geschlechtsspezifischen Unterschiede bzgl. des Verlaufs von Nieren- sowie deren Begleiterkrankungen und bearbeitet anschließend die Unterschiede in der medizinischen Versorgung in den verschiedenen Regionen der Welt.

Die niedrigeren $\mathrm{Hb}$-Level bei nierenkranken Frauen sind allgemein bekannt, finden aber in den nephrologischen Guidelines (noch) keine Beachtung. Auch sprechen Frauen häufiger schlechter auf ESA (Erythropoese stimulierende Agenzien) an. Bei Berechnung der notwendigen Dialysedosis mittels Kt/V wird diese für Frauen oft unterschätzt, da das Harnstoff-Verteilungs-Volumen (V) für Männer und Frauen gleichgesetzt wird. In der Gruppe der jüngeren Frauen ist die nicht kardiovaskuläre Mortalität (z. B. Infektionen) höher, Diabetes mellitus führt zu einem schlechteren Outcome bei weiblichen Patienten.

In vielen Regionen der Welt haben Frauen einen schlechteren Zugang zur medizinischen Versorgung als Männer. Neben wirtschaftlichen Verhältnissen spielt v.a. die soziokulturelle Stellung der Frau eine Rolle. In vielen Regionen Afrikas und der arabischen Welt sowie islamisch geprägten Gebieten erhalten i. d. R. meist männliche Patienten eine Nierenersatztherapie oder Nierentransplantation. Lateinamerika ist sehr heterogen bzgl. seiner ökonomischen Ressourcen und Ursachen für eine chronische Nierenerkrankung. In El Salva- dor erhält kaum jemand eine Nierenersatztherapie. Dagegen haben in den reicheren asiatischen Ländern alle Patienten gleichermaßen Zugang zur Nierenersatztherapie.

Doch auch in den DOPPS-Ländern (Europa, USA, Australien etc.) erhalten insbesondere ältere Frauen weniger Nierenersatztherapien als Männer, was v. a. an sozioökonomischen Faktoren, aber auch den Ärzten liegt. So initiieren sie die Dialyse bei Frauen häufiger erst bei einer niedrigeren GFR als bei Männern, vielleicht auch weil die Patientinnen weniger eindrucksvolle Symptome bieten. Frauen sterben häufiger als Männer im Prädialysestadium. In allen Weltregionen mit Ausnahme von Thailand sind Frauen häufiger Spender als Empfänger von Nierentransplantaten.

Im Hinblick auf die Therapie der chronischen bis hin zur terminalen Niereninsuffizienz sind Männer und Frauen biologisch verschieden. Dies sollte in den Behandlungsstrategien der Anämie, der Progressionsverlangsamung und der Dialysepflicht Beachtung finden. Diesbezüglich sind jedoch weitere Studien notwendig. Auffällig bleibt, dass Frauen häufiger als Männer Nieren spenden und seltener Transplantatnieren erhalten. Außerdem werden Frauen v. a. in ärmeren Ländern seltener einer lebensrettenden Nierenersatztherapie zugeführt.

\section{Kommentar}

Noch immer gibt es keine Gleichbehandlung der Frauen bzgl. der Nierenersatztherapie - auch in Deutschland [1]. Sie haben eine geringere Wahrscheinlichkeit, auf die Warteliste für die Nierentransplantation zu gelangen oder ein Lebendnierentransplantat zu erhalten. Auch haben sie eine höhere Wahrscheinlichkeit für einen späteren Dialysebeginn. Dialysepflichtige Frauen zeigen eine erhöhte Sterblichkeitsrate im Vergleich zu Männern, was auf eine Übersterblichkeit insbesondere jüngerer dialysepflichtiger Frauen und diabetischer
Dialysepatientinnen zurückzuführen ist. Weitere mit der Mortalität assoziierte Faktoren sind die zu geringe Dialysedosis v. a. durch eine Fehleinschätzung des HarnstoffVerteilungs-Volumens [2] und das Fehlen von funktionstüchtigen Dialysefisteln zu Dialysebeginn [3]. Die Ursachen für diese Phänomene sind heterogen, ihre Erforschung steht noch am Anfang.

\section{Korrespondenzadresse}

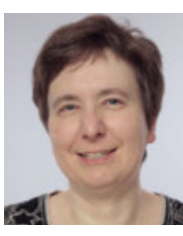

\section{Dr. med. Birgit Bader} Medizinische Klinik II St. Joseph Krankenhaus Berlin-Tempelhof GmbH Wüsthoffstr. 15 12101 Berlin birgit.bader@sjk.de

Literatur

[1] Hecking M, Bieber BA, Ethier ] et al. Sexspecific differences in hemodialysis prevalence and practice and the male-to-female mortality rate: the Dialysis Outcomes and Practice Patterns Study (DOPPS). PLoS Med 2014; 11: e1001750

[2] Daugirdas JT. We underdialyze women and smaller patients. Semin Dial 2016; 29: 303-305

[3] Stracke S, Fiene B, Koberstein P, Ernst F. Dialyse - Besonderheiten bei Frauen im Vergleich zu Männern. Dialyse aktuell 2012; 16: $180-184$ 